\title{
Aktuelle Empfehlungen zur Diagnostik der Riesenzellarteriitis
}

Thorsten Bley, Michael Zänker, Claudia Dechant, Nils Venhoff

\author{
Um schwere komplikative Verläufe wie Erblindung oder strukturelle Gefäßschäden \\ bei der Riesenzellarteriitis (RZA) zu vermeiden, bedarf es einer zeitnahen Diagnostik. \\ Dabei haben sich die Überwachung der Entzündungsparameter im Labor und ver- \\ schiedene bildgebende Verfahren sowie die histopathologische Beurteilung einer \\ Schläfenarterienbiopsie etabliert. Auf Basis aktueller Empfehlungen werden die \\ diagnostischen Verfahren im Folgenden diskutiert.
}

\section{Einführung}

Die Riesenzellarteriitis (RZA) manifestiert sich typischerweise jenseits des 50. Lebensjahres mit steigender Prävalenz und Inzidenz im Alter und einem Gipfel um das 75. Lebensjahr [1]. Die Erkrankung hat ein starkes Nord-Süd-Gefälle mit hohen Inzidenzraten von 27 - 56/ 100000 in Skandinavien, gegenüber 3,5/100 000 in Deutschland [1, 2]. Kaukasier sind häufiger betroffen als andere Ethnien. Frauen sind 2-3-mal häufiger betroffen als Männer.

\section{Klinik}

Die vielfältigen Symptome der RZA lassen sich größtenteils auf Folgendes zurückführen:

1. die lokale Inflammation der Gefäßwand,

2. die Gewebsischämie infolge stenosierender Prozesse und

3. die systemische Inflammation ( $\triangleright$ Abb. 1).

Die systemische Entzündungsreaktion führt bei der Mehrheit der Patienten (40-100\%) zu allgemeinem Krankheitsgefühl bis hin zu Fieber, Nachtschweiß und Gewichtsverlust [2, 3]. Bei rund $50 \%$ der Patienten finden sich typische Beschwerden einer Polymyalgia rheumatica (PMR) mit Morgensteifigkeit und proximal betonten Myalgien im Schultergürtel- und Beckenbereich [2 -4].

Klassisch und ursprünglich namengebend ist die Manifestation im Kopfbereich. Bei $75 \%$ der Patienten treten klinisch gut erkennbare Entzündungen der oberflächlichen Schläfenarterienäste (A. temporalis superficialis) auf. Der Terminus „Arteriitis temporalis“ sollte dennoch nicht mehr benutzt werden, da nicht bei allen RZA-Patienten die A. temporalis (TA) betroffen ist.

Typisch für diese kraniale Form der RZA sind plötzlich neu aufgetretene, besonders stark empfundene und nicht selten Analgetika-refraktäre Kopfschmerzen, die temporal oder okzipital lokalisiert sind oder ein diffuses Verteilungsmuster haben [2-4]. Bei der klinischen Untersuchung findet sich oft eine druckdolente TA, die gerötet und knotig geschwollen sein kann und eine verminderte Pulsatiliät aufweist.

Gezielt sollten eine Überempfindlichkeit der Kopfhaut, Schmerzen beim Kämmen sowie eine beim Kauen auftretende, ischämiebedingte Kiefer- oder Zungenclaudicatio erfragt werden. Nekrosen der Kopfhaut treten nur sehr selten auf. Etwa 3-4\% der Patienten entwickeln zerebrale Infarkte aufgrund der Beteiligung des Vertebralis-, Basilaris- oder Karotisstromgebiets [2].

\section{Merke \\ Ein absoluter Notfall ist die Augenbeteiligung, die bei $12-40 \%$ der Patienten auftritt $[2,5]$.}

Als Notfälle sind ein plötzlicher, schmerzloser und meistens einseitiger Visusverlust, vorübergehende Erblindung (Amaurosis fugax) und/oder Gesichtsfeldausfall zu betrachten [2, 5]. Die häufigste Ursache ist eine anteriore ischämische Optikusneuropathie (AION) als Folge eines entzündungsbedingten Verschlusses der posterioren Ziliararterien. Die Ischämie der extraokulären Augenmuskeln oder der versorgenden Hirnnerven kann zu Doppelbildern führen, die - im Gegensatz zu eingetretener Erblindung - unter Therapie in der Regel reversibel sind. Ziel der raschen Therapie-Initiierung ist, die Erblindung des 2. Auges zu verhindern, die ohne Therapie bei $60 \%$ der Patienten innerhalb weniger Tage auftritt [2].

Bei isolierter Manifestation der RZA an der Aorta und ihren Abgängen (ohne Beteiligung der Kopfgefäße) können Patienten sich durchaus beschwerdearm mit nur unspezifischen Symptomen und Entzündungskonstellation präsentieren. Je nach Kohorte und verwendeter diagnostischer Methode (CT-Angiografie, farbkodierte Duplex- 


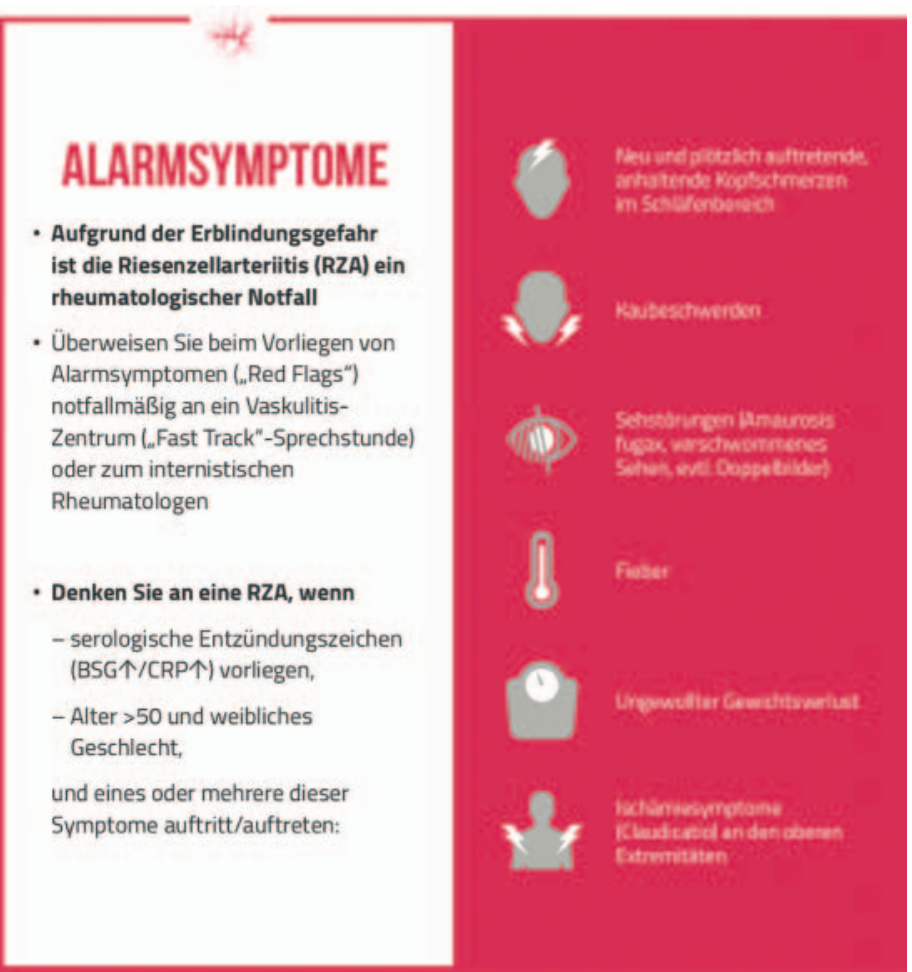

- Abb. 1 Alarmsymptome („Red Flags“) für eine RZA. Da bei bis zu $50 \%$ der RZA-Patienten polymyalgische Beschwerden auftreten, sollte auch bei PMR-Symptomatik an eine RZA gedacht und diese ausgeschlossen werden! Quelle: Chugai Pharma Europe Ltd.

Sonografie, FDG-PET-CT) schwankte die Inzidenz zwischen 22,7 und $83 \%$ [4].

Gefäßstenosen können zu lokalen Strömungsgeräuschen sowie seitendifferenten Blutdruck- und Pulsatilitätsbefunden führen, sodass die Gefäße stets auskultiert, beidseitig der Blutdruck gemessen und der Radialispuls palpiert werden sollten. Höhergradige Stenosen können über Claudicatio und Ruheschmerzen bis zur Gangrän von Fingern und der Hand führen.

Insgesamt ist die Mortalität bei RZA-Patienten gegenüber der Normalbevölkerung nicht erhöht. Eine Ausnahme stellen Patienten mit Aortenaneurysma dar. Insbesondere thorakale Aortenaneurysmata sind 17-mal häufiger als in der Allgemeinbevölkerung und treten bei knapp $20 \%$ der RZA-Patienten auf. Sie sind insbesondere durch das Dissektionsrisiko mit einer 5,1-fach erhöhten Mortalität assoziiert [5].

\section{Diagnostik}

\section{Labor}

Ein spezifischer Labormarker für die RZA ist bislang nicht verfügbar. Bei ca. $90 \%$ histologisch gesicherter RZA liegen zum Diagnosezeitpunkt jedoch erhöhte Blutkörperchen- senkungsgeschwindigkeit (BKS) und/oder erhöhtes C-reaktives Protein vor. Diese Werte werden im klinischen Alltag auch zur Überwachung der Krankheitsaktivität und im klinischen Kontext zur Therapiesteuerung genutzt. Patienten mit späteren RZA-Rezidiv weisen im Vergleich zu Patienten mit rezidivfreien Verläufen höhere initiale Werte von BKS, Akut-Phasen-Proteinen und Leukozyten auf. Auch Anämie und Thrombozytose finden sich häufig bei aktiver RZA. Die Bestimmung von Auto-Antikörpern dient ausschließlich differenzialdiagnostischer Abgrenzung.

\section{Temporalarterienbiopsie (TAB)}

Der histopathologische Nachweis eines Infiltrats mit prädominant mononukleären Zellen und mehrkernigen Riesenzellen im Biopsat der oberflächlichen Temporalarterie gilt als beweisend für die Diagnose der RZA. Allerdings gibt es unterschiedliche histologische Muster bei der RZA, was vermutlich den verschiedenen Phasen der Entzündung und des Schadens entspricht [6]. Eine allgemeingültige Definition einer positiven Biopsie existiert nicht. Generell werden folgende Merkmale für positiv gewertet:

- transmurale Entzündung mit Riesenzellen

- Entzündung am Übergang zwischen Adventitia und Muscularis media

- Fragmentierung der Lamina elastica interna

Wenn das Ergebnis nicht eindeutig ist, kann ein Referenzpathologe hinzugezogen werden. Die TAB lässt sich in Lokalanästhesie durchführen, aufgrund des segmentalen Befalls wird eine Biopsatlänge von mind. 0,5-1 cm empfohlen.

Eine bereits 2-4 Wochen vor der Biopsie eingeleitete Glukokortikoid-Therapie (GC) beeinflusst die Rate eines positiven TAB-Befundes unwesentlich [7]. Die diagnostische Wertigkeit der TAB wird durch die niedrige Sensitivität limitiert, die vor Kurzem in der TABUL-Studie mit 39\% bestätigt wurde [8]. Die hohe Rate falsch negativer Befunde bei der TAB lässt sich unter anderem durch einen für die RZA typischen segmentalen vaskulitischen Befall erklären.

Merke

Der histopathologische Nachweis eines Infiltrats mit prädominant mononukleären Zellen und mehrkernigen Riesenzellen im Biopsat der oberflächlichen Temporalarterie gilt als beweisend für die Diagnose der RZA.

\section{Bildgebende Diagnostik}

Als bildgebende Verfahren haben sich die farbkodierte Duplex-Sonografie (FKDS), die hochauflösende Magnetresonanztomografie (MRT), die Computertomografie (CT) bzw. CT-Angiografie (CTA) und die ${ }^{18} \mathrm{~F}$-Fluordeoxyglukose-Positronenemissionstomografie mit CT (FDGPET-CT) etabliert. 
Gemäß den kürzlich veröffentlichten Empfehlungen der European League Against Rheumatism (EULAR) zur Anwendung der bildgebenden Verfahren wird bei Patienten mit Verdacht auf eine RZA eine frühzeitige Bildgebung in der Hand erfahrener Untersucher komplementär zu den klinischen Kriterien für die Diagnosestellung der RZA angeraten [9]. Die GC-Behandlung führt dazu, dass die Zeichen der aktiven Entzündung in der Bildgebung innerhalb weniger Tage schwächer werden und dadurch falsch negative Befunde entstehen können. Daher soll die Bildgebung so früh wie möglich initiiert werden und auf keinen Fall den Beginn der Therapie verzögern.

Bei begründetem klinischem Verdacht auf eine RZA und positiver Bildgebung aus der Hand eines erfahrenen Untersuchers kann von weiteren diagnostischen Bemühungen, wie z. B. einer Biopsie der oberflächlichen Temporalarterie, abgesehen werden [9]. Die EULAR-Empfehlungen argumentieren nicht gegen eine Biopsie der oberflächlichen Temporalarterie. Allerdings wird empfohlen, eine Bildgebung in Vaskulitis-Zentren mit hoher Expertise in der Vaskulitis-Bildgebung als initialen Test durchzuführen, da diese nicht invasiv ist, ein größeres Gefäßterritorium abdeckt und zu einer geringeren Zahl falsch negativer Ergebnisse führen kann.

Die frühzeitige Detektion einer Aortitis ist relevant, da das Risiko einer Aortendilatation im weiteren Verlauf der Erkrankung bei initialem Aortenbefall mit einem Hazard Ratio von 3,16 in einer großen aktuellen Studie erhöht war [10].

\section{Merke}

Bei Patienten mit Verdacht auf RZA wird eine frühzeitige Bildgebung komplementär zu den klinischen Kriterien für die Diagnosestellung der RZA angeraten, ohne dabei den Beginn der Therapie zu verzögern.

\section{Farbkodierte Duplex-Sonografie (FKDS)}

Durch die richtungsweisenden Arbeiten von Schmidt et al. [11] wurde die farbkodierte Duplex-Sonografie (FKDS) zum ersten Schnittbildverfahren, das zur Diagnostik einer RZA beitragen konnte. Die FKDS kann, neben Gefäßlumen und Blutfluss, auch Dicke und Struktur der Gefäßwände darstellen.

Derzeit empfohlene Linearschallköpfe mit > $15 \mathrm{MHz}$ verfügen, bei einer lateralen und axialen Ortsauflösung von $0,1 \mathrm{~mm}$, über eine höhere Auflösung als die MRT und CT. Sie ermöglichen eine optimale Beurteilung auch der parietalen und frontalen TA-Äste mit gerade noch 0,7 $0,9 \mathrm{~mm}$ systolischem Lumen und Wanddicken von 0,7 mm [10] ( Tab. 1). Power-Doppler und B-Flow-Sonografie ermöglichen inzwischen auch die Darstellung langsamer Flüsse in kleinen Gefäßen mit hoher Ortsauflösung
- Tab. 1 Empfehlungen für technische und operationale Parameter der Schnittbildgebung bei der Diagnose der RZA.

\begin{tabular}{|c|c|}
\hline Bildgebungsverfahren & Untersuchungsparameter \\
\hline \multirow[t]{2}{*}{$\begin{array}{l}\text { Farbkodierte } \\
\text { Duplex-Sonografie } \\
\text { (FKDS) }\end{array}$} & $\begin{array}{l}\text { B-Mode } \\
\text { " } \geq 15 \mathrm{MHz} \text { für TA-Untersuchung } \\
\text { - } 7-15 \mathrm{MHz} \text { für extrakranielle supraaortale } \\
\text { Arterien } \\
\text { " } \text { Bildtiefe: } \\
-10-20 \mathrm{~mm} \text { für TA-Untersuchung } \\
-30-40 \mathrm{~mm} \text { für extrakranielle supraaortale } \\
\quad \text { Arterien }\end{array}$ \\
\hline & $\begin{array}{l}\text { Doppler } \\
\text { - } 7-12 \mathrm{MHz} \text { für TA-Untersuchung } \\
\text { - } 4-8 \mathrm{MHz} \text { für extrakranielle supraaortale } \\
\text { Arterien } \\
\text { - } \text { optimale Pulswiederholungsfrequenz (PRF) } \\
\text { - } 2-3 \mathrm{kHz} \text { für TA-Untersuchung } \\
\text { - } 3-4 \mathrm{kHz} \text { für extrakranielle supraaortale } \\
\text { Arterien } \\
\text { - Einstellung Schalleintrittswinkel } \leq 60^{\circ} \text { zur } \\
\text { Gefäßachse }\end{array}$ \\
\hline MRT & $\begin{array}{l}\text { " T1w Spin-Echo-Sequenz (TR/TE 500/22 ms), } \\
\text { räumliche Auflösung } 195 \times 260 \mu \mathrm{m}^{2} \text {, Schicht- } \\
\text { dicke } 3 \mathrm{~mm} \\
\text {. Kopfspule } \geq 8 \text { Kanäle, transversale Schichtfüh- } \\
\text { rung parallel zum Corpus callosum }\end{array}$ \\
\hline MRA & $\begin{array}{l}\text { - } 3 \text { D Turbo-Spin-Echo (TR/TE 1000/35 ms), } \\
\text { räumliche Auflösung } 1,2 \times 1,3 \times 2 \mathrm{~mm}^{3} \\
\text { - Kombination von Kopf/Hals- und Körperspulen } \\
\text { - koronare Schichtführung adduzierter Arme zur } \\
\text { Darstellung der A. axillaris und proximalen } \\
\text { A. brachialis }\end{array}$ \\
\hline FDG-PET & $\begin{array}{l}\text { - Datenakquisition: } 90 \text { min (mind. } 60 \text { min) nach } \\
\text { Applikation von FDG } \\
\text {. Blutglukosewert < } 7 \text { mmol/l (126 mg/dl), } \\
\text { Minimum < } 10 \mathrm{mmol} / / \text { ( } 180 \mathrm{mg} / \mathrm{dl}) \\
\text {. am Tag der Untersuchung morgens keine } \\
\text { GC-Gabe, damit zur Untersuchung der Glukose- } \\
\text { stoffwechsel nicht gestört ist; nach Durchfüh- } \\
\text { rung des PET kann GC gegeben werden }\end{array}$ \\
\hline CT und CTA & $\begin{array}{l}\text { - Datenakquisition: Kollimation von 0,6 mm, } \\
\text { rekonstruierte Schichtdicke } \leq 1 \mathrm{~mm} \\
\text { - für EKG-getriggerte Akquisition der CTA: } 60 \text { - } \\
120 \mathrm{ml} \text { jodhaltiges Kontrastmittel (adaptiert an } \\
\text { Körpergewicht des Patienten) mit Applikations- } \\
\text { geschwindigkeit von } 4 \mathrm{ml} / \mathrm{s} \\
\text { - ca. } 50 \text { sec später folgt Scan in der venösen } \\
\text { Phase zur besseren Abgrenzung der Gefäß- } \\
\text { wand von dem Gefäßlumen }\end{array}$ \\
\hline
\end{tabular}

und Gefäßwandabgrenzung, unabhängig vom Anschallwinkel und ohne Überstrahlungs- und Aliasing-Artefakte.

Die FKDS ist breit verfügbar, relativ kostengünstig, nicht invasiv, frei von Strahlung und kommt ohne jodhaltige Kontrastmittel aus. Gleichwohl sind gezielte Ausbildung und Erfahrung des Untersuchers erforderlich, um eine hohe Interobserver-Reliabilität zu erreichen [12]. Geübten Untersuchern gelingt innerhalb von 15 Minuten die 


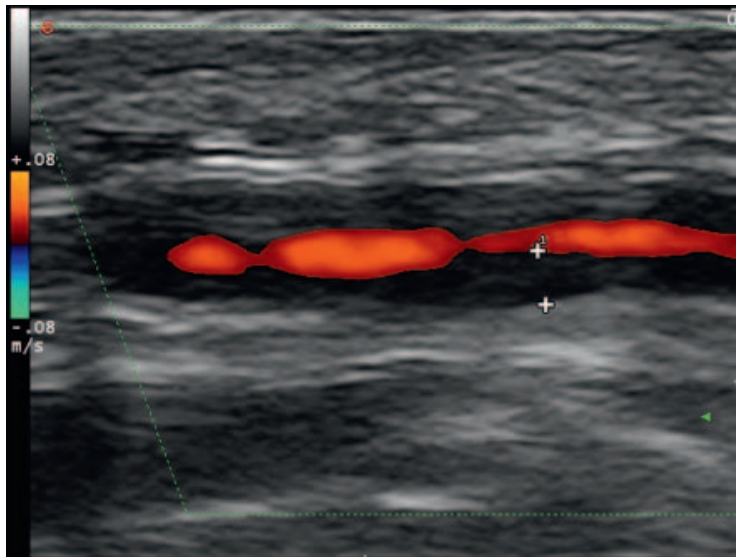

Abb. 2 Befund farbkodierte Duplex-Sonografie: HaloZeichen $\operatorname{der}$ A. temporalis superficialis bei Riesenzellarteriitis (RZA). Quelle: Prof. W. Schmidt, Rheumaklinik BerlinBuch.

beidseitige Untersuchung der TA, inklusive ihrer parietalen und temporalen Äste sowie beider Aa. axillares.

Entzündliche Wandverdickungen im Rahmen der RZA führen in Querschnitt- und Längsschnittbildern zu homogenen und echoarmen konzentrisch verdickten Wandabschnitten um ein zentrales Flusssignal, dem sogenannten Halo-Zeichen ( $\triangleright$ Abb. 2).

Bereits 1997 ermittelten Schmidt et al. für das Halo-Zeichen eine Sensitivität von $73 \%$ und Spezifität von $100 \%$ [11]. Bis 2010 lagen 3 Metaanalysen mit 49 inkludierten Studien und 3636 Patienten vor. Für unilaterale Halos fanden sich gepoolte Sensitivitäten von 55 - $75 \%$ und Spezifitäten von $82-94 \%$, je nach Kohorte und klinisch oder histologisch gesicherter Diagnose [13, 14]. Ein bilateraler Halo-Nachweis hat eine Spezifität von $100 \%$. Ein weiteres Zeichen ist die verminderte Komprimierbarkeit des Halo durch leichten Druck mit dem Schallkopf, welches eine hohe Sensitivität (73\%) und Spezifität (100\%) bei höchster Übereinstimmungsrate $(\alpha=0,92)$ der Untersucher aufweist [15]. Bei der RZA ebenfalls nachweisbare, entzündliche Wandstenosen und okkludierte Gefäße tragen wegen geringer Spezifität nicht zu einer höheren diagnostischen Wahrscheinlichkeit bei $[11,13]$.

Zudem kommt es zum Verlust der 3-schichtigen Echodarstellung sowie zur Zunahme der Intima-Media-Dicke (IMT) der betroffenen arteriellen Abschnitte. Eine IMT $\operatorname{der}$ A. temporalis communis $>0,42 \mathrm{~mm}$, der Frontaläste $>0,34 \mathrm{~mm}$ und der Parietaläste $>0,29 \mathrm{~mm}$ wurden als Cut-offs mit hoher Sensitivität und Spezifität für eine RZA ermittelt ( Tab. 2) [15].

Als extrakraniale Gefäßmanifestationen sind am häufigsten die Aa. axillares und subclaviae betroffen, überwiegend bilateral und in $50 \%$ mit Stenosen einhergehend
[16]. Bei der A. axillaris weisen Halo und IMT >1,0 mm mit hoher Sensitivität und Spezifität auf eine RZA hin [15].

Prinzipiell können viele Gefäßabschnitte beteiligt sein. Da in den meisten Fällen gleichzeitig die Aa. axillares betroffen sind, wird die initiale Untersuchung von Temporal- und Axillararterien empfohlen. Bei Nachweis einer extrakranialen RZA sollte die Diagnostik mittels Schnittbildverfahren (CTA, MRA, PET), je nach lokaler Expertise und Verfügbarkeit, ergänzt werden. Bei bis zu 20 \% der Patienten mit Großgefäßvaskulitis sind Aneurysmen überwiegend der Aorta ascendens und des Aortenbogens vorhanden, die sonografisch nur durch transösophageale Techniken (TEE, EUS) nachweisbar sind.

Im klinischen Alltag kann bei typischer Symptomatik und Befundkonstellation sowie nachgewiesenem Halo auf eine zusätzliche TAB verzichtet werden, da die bereits hohe Spezifität durch eine positive TAB kaum gesteigert werden kann. Außerdem reduziert eine negative TAB wegen geringer Sensitivität die Wahrscheinlichkeit einer RZA kaum. Sinnvoll ist die TAB jedoch bei unspezifischer Sonografie, aber typischer Klinik und therapeutischer Relevanz einer positiven Histologie. Grundlage hierfür sind histologische Subsets der Temporalarteriitis mit alleiniger Kleingefäßvaskulitis im Bereich der Adventitia oder der Vasa vasorum. Diese können ebenso zu kranialen ischämischen Ereignissen führen und sich jedoch ohne transmurale Entzündung der FKDS entziehen [17]. Die Ausbeute der TAB kann durch vorherige Sonografie und Markierung geeigneter Areale verbessert werden.

Halos können sich unter Therapie zurückbilden und verschwinden in $95 \%$ der Fälle innerhalb weniger Tagen bis einiger Monate. Die Sensitivität der FKDS kann 2 Tage nach GC-Beginn nur noch $50 \%$ betragen [8]. Dagegen fand sich eine Persistenz des Halos über 6 Monate trotz klinischer und serologischer Remission [18]. Persistierende Gefäßveränderungen korrelieren mit dem Ausmaß des Entzündungsprozesses, der Höhe der Entzündungsmarker sowie dem Durchmesser der betroffenen Gefäße und mit extrakranieller Manifestation [19].

Durch den Einsatz der kontrastverstärkten Sonografie (CEUS) wird gegenwärtig versucht, die vermehrte Kontrastierung innerhalb der Gefäßwände durch Neovaskularisation mit der Entzündungsaktivität der RZA zu korrelieren [20]. Künftige Studien müssen die Eignung des Verfahrens für die Verlaufsbeurteilung der RZA noch klären.

Merke

Der nicht komprimierbare Halo ist bei der FKDS in der Hand eines erfahrenen Untersuchers das bildgebende Kriterium der 1. Wahl in der Diagnostik der RZA. 
> Tab. 2 Intima-Media-Dicken der A. temporalis und A. axillaris (Normwerte und Cut-off-Werte für die Diagnostik einer Riesenzellarteriitis).

\begin{tabular}{|c|c|c|c|c|c|}
\hline Arterielle Abschnitte & $\begin{array}{l}\text { Kontrollen } \\
\mathrm{n}=\mathbf{4 0} \\
\text { IMT }(\mathrm{mm})\end{array}$ & $\begin{array}{l}\text { Vaskulitis } \\
n=40 \\
\text { IMT }(\mathrm{mm})\end{array}$ & Cut-off-IMT (mm) & Sensitivität (\%) & Spezifität (\%) \\
\hline A. temporalis communis & $0,23 \pm 0,04$ & $0,65 \pm 0,18$ & 0,42 & 100 & 100 \\
\hline Ramus frontalis & $0,19 \pm 0,03$ & $0,54 \pm 0,18$ & 0,34 & 100 & 100 \\
\hline Ramus parietalis & $0,20 \pm 0,03$ & $0,50 \pm 0,17$ & 0,29 & 97,2 & 98,7 \\
\hline A. axillaris & $0,59 \pm 0,10$ & $1,72 \pm 0,41$ & 1,0 & 100 & 100 \\
\hline
\end{tabular}

\section{Magnetresonanztomografie (MRT)}

Die hochauflösende MRT kann mit einer hohen räumlichen Auflösung die muralen Entzündungszeichen wie Wandverdickung $>600 \mu \mathrm{m}$ und vermehrte Kontrastmittelaufnahme der oberflächlichen Temporalarterien präzise und untersucherunabhängig darstellen [21, 22]. In den EULAREmpfehlungen wird die MRT bei inkonklusivem FKDSBefund oder Diskonkordanz zur klinischen Symptomatik als alternative Bildgebung empfohlen ( Tab. 1) [9].

Sowohl bei 1,5 Tesla als auch bei 3 Tesla gelingt eine exakte Darstellung der Gefäßwand der oberflächlichen Schläfen- und Hinterhauptsarterien [21 - 24] ( Abb.3). Die höhere Magnetfeldstärke ermöglicht eine bessere Signalausbeute und höhere Bildqualität. Ein direkter, intra-individueller Vergleich von 7 Tesla und 3 Tesla-Untersuchungen bei Patienten mit RZA konnte diese Erkenntnis bestätigen, da die muralen Entzündungszeichen bei der höheren Feldstärke deutlich besser erkannt werden konnten.

Die native T2-gewichtete MRT-Bildgebung ist der T1-gewichteten, Kontrastmittel-unterstützten Technik unterlegen und kann bei schwach ausgeprägten Entzündungszeichen falsch negative Ergebnisse liefern [25].

Die vaskulitischen MRT-Zeichen werden unter erfolgreicher GC-Therapie sehr schnell schwächer und verschwinden bei Remission vollständig. Daher sollte die Bildgebung vorzugsweise innerhalb der ersten 5 Tage nach Therapiebeginn erfolgen [26]. Die hochauflösende MRT kann nicht nur die entzündlichen Wandveränderungen der extraduralen kraniellen Arterien, sondern auch der intrakraniellen, intraduralen Arterien nachweisen [27].

In Kombination mit einer MR-Angiografie der Aorta und ihrer Äste kann die murale Entzündung der oberflächlichen Kopfarterien einschließlich des kraniellen Befallsmusters erfasst und gleichzeitig die Beteiligung der extrakraniellen großen Arterien evaluiert werden. Es wird die kombinierte Verwendung von Kopf-, Hals- und
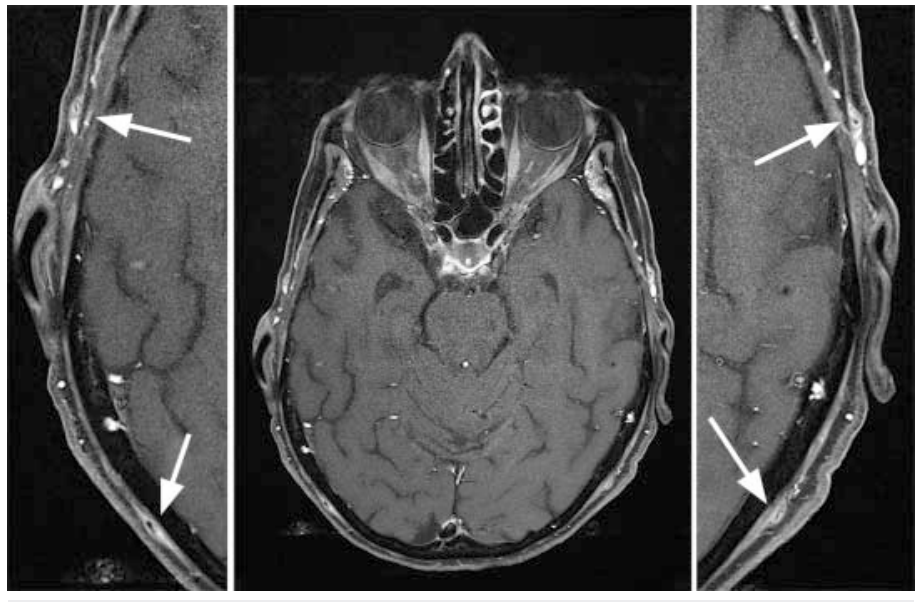

- Abb. 3 3T-MRT einer 77-jährigen RZA-Patientin mit bilateraler Beteiligung (frontal und okzipital).

Körperspulen angeraten. Dabei kann eine Gefäßdarstellung von der Karotisbifurkation bis zu den Oberbaucharterien, bzw. vorzugsweise bis zu den Beckenarterien, erreicht werden, um mögliche Stenosen, Verschlüsse, Aneurysmata oder Dissektionen zu detektieren. Die koronare Schichtführung bei adduzierten Armen erlaubt dabei eine bilaterale Darstellung der A. axillaris und proximalen A. brachialis. Mit der sogenannten „Black blood“Technik wird ein hoher Kontrast zwischen dem signallosen „schwarzen“ Lumen und der signalreichen, in den entzündeten Segmenten stark kontrastierten und damit besonders „weißen“ Arterienwand, erreicht.

\section{Merke}

Die hochauflösende MRT der oberflächlichen Kopfarterien kann die muralen Entzündungszeichen der oberflächlichen Kopfarterien darstellen und wird bei inkonklusivem FKDS-Befund oder Diskonkordanz zur klinischen Symptomatik als Alternative angeraten. 


\section{Fluordeoxyglukose- Positronenemissions- tomografie (FDG-PET)}

Die FDG-PET wird als das sensitivste Verfahren zur Darstellung auch geringer entzündlicher Aktivität angesehen, da selbst eine FDG-Aufnahme im pikomolaren Bereich in der FDG-PET dargestellt werden kann [28]. Gleichzeitig bildet die FDG-PET den Gefäßbaum von den zervikalen Arterien bis hin zu den Extremitätenarterien ab, sodass die extrakranielle Großgefäßbeteiligung sehr sensitiv beurteilt werden kann ( $\bullet$ Tab. 1).

Schwierig kann die Unterscheidung von vaskulitischen Läsionen und entzündlich veränderten arteriosklerotischen Plaques, insbesondere bei den Extremitätenarterien, sein. Die Bewertung der Bilder erfolgt qualitativ, da noch kein standardisiertes Verfahren zur objektiven Beurteilung der entzündlichen Aktivität mittels FDG-PET existiert [29]. Die physiologische Glukoseaufnahme variiert je nach Gefäßterritorium [30].

Die Verfügbarkeit einer FDG-PET ist eingeschränkt und der finanzielle Aufwand nicht unerheblich. Die FDG-PET wird für die Darstellung entzündlicher Veränderungen der oberflächlichen Kopfarterien derzeit nicht empfohlen, da diese aufgrund des starken Glukosestoffwechsels des ZNS in der FDG-PET überlagert werden. Zudem erlaubt die räumliche Auflösung der aktuell verbreiteten PET-Scanner zumeist keine klare Abgrenzung dieser kleinen Gefäße.

\section{Merke}

Die FDG-PET ist das wohl sensitivste Verfahren zur Darstellung der Entzündungsaktivität und des Befallsmusters.

\section{Computertomografie (CT) und CT-Angiografie (CTA)}

Die CT und CTA sind weit verbreitet verfügbar, schnell durchführbar und in der Datenakquisition standardisiert und somit weniger untersucherabhängig ( $>$ Tab. 1). Allerdings setzt die zur Beurteilung einer möglichen muralen Entzündungsaktivität der Aorta und ihrer großen Äste notwendige intravenöse Kontrastmittelgabe eine gute Nierenfunktion voraus.

Die arterielle Phase ist wichtig für die exakte Lumenbeurteilung der Arterien. Die Verzögerung zur venösen Phase ist wichtig, um Zeit für die entzündliche Kontrastmittelanreicherung der betroffenen Gefäßwand über die Vasa vasorum zu geben und gleichzeitig diese Zeit für die Abschwächung der in der arteriellen Phase initial hohen Kontrastierung des Gefäßlumens zu nutzen. Dadurch ergibt sich in der venösen Phase ein besserer Kontrast zwischen der entzündeten und somit sehr dichten Gefäßwand und dem weniger kontrastierten Blut in dem nicht mehr so dichten Gefäßlumen.
Der Einsatz von Röntgenstrahlen ist ein Nachteil der CT und CTA, der jedoch durch den diagnostischen Nutzen der Untersuchung bei korrekter Indikationsstellung gerechtfertigt ist.

\section{KERNAUSSAGEN}

- Die aktuellen EULAR-Empfehlungen beinhalten eine frühzeitige Bildgebung zur Komplettierung der klinischen Beurteilung, ohne die Therapie zu verzögern.

- Die farbkodierte Duplex-Sonografie (FKDS) der oberflächlichen Kopfarterien und $\operatorname{der}$ A. axillaris wird in der Hand eines geübten Untersuchers als Bildgebung der 1. Wahl bei RZA empfohlen.

- Bei fehlender Verfügbarkeit oder inkonklusivem Befund der FKDS wird die hochauflösende MRT angeraten, die sowohl murale Entzündungszeichen der oberflächlichen Temporalarterien als auch eine Beteiligung der großen Körperarterien bzw. das Vorliegen eines Aortenaneurysma darstellen kann.

- Die FDG-PET wird als sensitivstes Verfahren zur Beurteilung einer entzündlichen Beteiligung der großen extrakraniellen Arterien angesehen.

- Bei klinischem Verdacht auf RZA und positiver Bildgebung kann von einer Biopsie der oberflächlichen Temporalarterie abgesehen werden.

\section{Interessenkonflikt}

Alle Autoren sind Mitglieder des GCA-Experten-Board von Roche/Chugai.

TB: Forschungsförderung durch die Deutsche Forschungsgemeinschaft, Mitglied Advisory Board/Beratertätigkeit: Siemens Healthineers, Vortragstätigkeit: Guerbet, HeartFlow, Fa. Roche/Chugai, Siemens.

MZ: Tätigkeit in wissenschaftlichen Advisory Boards der Firmen Roche, MSD und Celgene sowie Vortragshonorare von Roche, MSD und Celgene.

CD: Vortragshonorare der Roche Pharma AG, AbbVie, Novartis, medac, Boehringer Ingehlheim, Pfizer, Actelion. NV: Vortrags-/Beratertätigkeiten: Fa. Roche, Fa. Chugai, Bristol-Myers-Squibb, Pfizer, Novartis, AbbVie, UCB, Celgene, Medac.

\section{Autorinnen/Autoren}

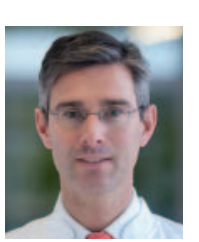

Univ.-Prof. Dr. med. Thorsten Bley

Direktor des Instituts für Diagnostische und Interventionelle Radiologie, Universitätsklinikum Würzburg. 


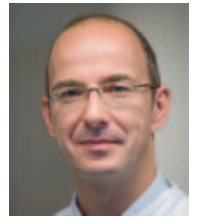

\section{Dr. med. Michael Zänker}

Chefarzt Abteilung Innere Medizin mit Schwerpunkten Gastroenterologie, Nephrologie und Rheumatologie am Immanuel Klinikum Bernau Herzzentrum Brandenburg. Medizinische Hochschule Brandenburg.

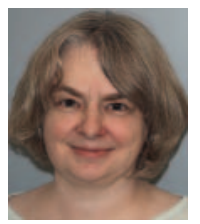

\section{Dr. med. Claudia Dechant}

Oberärztin, Rheumaeinheit, Medizinische Klinik und Poliklinik IV, Klinikum der Universität München.

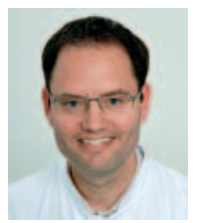

\section{PD Dr. med. Nils Venhoff}

Geschäftsführender Oberarzt, Klinik für Rheumatologie und Klinische Immunologie, Vaskulitis-Zentrum Freiburg, Universitätsklinikum Freiburg.

\section{Korrespondenzadresse}

Prof. Dr. med. Thorsten A. Bley

Universitätsklinikum Würzburg

Institut für Diagnostische und Interventionelle Radiologie

Oberdürrbacher Straße 6

97080 Würzburg

bley_t@ukw.de

\section{Danksagung}

Die Autoren sind Mitglieder des von den Firmen Roche Pharma AG/Chugai Pharma Europe Ltd. initiierten Experten-Board für Riesenzellarteriitis und danken Frau Dr. Recker für die redaktionelle Überarbeitung des Manuskriptes sowie den beiden Firmen für die Übernahme der open access Publikationsgebühren.

\section{Literatur}

[1] Mohammad AJ, Nilsson JA, Jacobsson LT et al. Incidence and mortality rates of biopsy-proven giant cell arteritis in southern Sweden. Ann Rheum Dis 2015; 74: $993-997$

[2] Ness T, Bley TA, Schmidt WA et al. Diagnose und Therapie der Riesenzellarteriitis. Dtsch Ärzeblatt 2014; 111: 1 - 12

[3] Hoffman GS. Giant Cell Arteritis. Ann Intern Med 2016; 165: $65-80$

[4] Hellmich B. Management der Polymyalgia rheumatica und der Großgefäßvaskulitiden. Internist 2016; 57: 1069-1078

[5] Kermani TA, Warrington KJ, Crowson CS et al. Large-vessel involvement in giant cell arteritis: a population-based cohort study of the incidence-trends and prognosis. Ann Rheum Dis 2013; 72: $1989-1994$

[6] Hernández-Rodríguez ], Murgia G, Villar I et al. Description and Validation of Histological Patterns and Proposal of a Dynamic Model of Inflammatory Infiltration in Giant-cell Arteritis. Medicine (Baltimore) 2016; 95: e2368. doi:10.1097/ MD.0000000000002368

[7] Narváez J, Bernad B, Roig-Vilaseca D et al. Influence of previous corticosteroid therapy on temporal artery biopsy yield in giant cell arteritis. Semin Arthritis Rheum 2007; 37: 13-19
[8] Luqmani R, Lee E, Singh S et al. The Role of Ultrasound Compared to Biopsy of Temporal Arteries in the Diagnosis and Treatment of Giant Cell Arteritis (TABUL): a diagnostic accuracy and cost-effectiveness study. Health Technol Assess 2016; 20: 1-238

[9] Dejaco C, Ramiro S, Duftner C et al. EULAR recommendations for the use of imaging in large vessel vasculitis in clinical practice. Ann Rheum Dis 2018; 77: 636-664

[10] de Boysson H, Daumas A, Vautier M et al. Large-vessel involvement and aortic dilation in giant-cell arteritis. A multicenter study of 549 patients. Autoimmun Rev 2018; 17: 391 - 398

[11] Schmidt WA, Kraft HE, Vorpahl K et al. Color Duplex Ultrasonography in the Diagnosis of Temporal Arteritis. N Engl J Med 1997; 337: $1336-1342$

[12] Chrysidis S, Duftner C, Dejaco C et al. Definitions and reliability assessment of elementary ultrasound lesions in giant cell arteritis: a study from the OMERACT large Vessel Vasculitis Ultrasound Working group. RMD Open 2018; 4: e000598. doi:10.1136/ rmdopen-2017-000598

[13] Arida A, Kyprianou M, Kanakis M et al. The diagnostic value of ultrasonography-derived edema of the temporal artery wall in giant cell arteritis: a second meta-analysis. BMC Musculoskelet Disord 2010; 11: 44

[14] Karassa FB, Matsagas MI, Schmidt WA et al. Meta-analysis: test performance of ultrasonography for giant-cell arteritis. Ann Intern Med 2005; 142: 359-369

[15] Schäfer VS, Juche A, Ramiro S et al. Ultrasound cut-off values for intima-media thickness of temporal, facial and axillary arteries in giant cell arteritis. Rheumatoly (Oxford) 2017; 56: 479-1483

[16] Schmidt WA, Seifert A, Gromnica-Ihle E et al. Ultrasound of proximal upper extremity arteries to increase the diagnostic yield in large-vessel giant cell arteritis. Rheumatology 2008; 47: 96-101

[17] Muratore F, Boiardi L, Restuccia G et al. Comparison between colour duplex sonography findings and different histological patterns of temporal artery. Rheumatoly (Oxford) 2013; 52 : $2268-2274$

[18] Pérez López J, Solans Laqué R, Bosch Gil JA et al. Colour-duplex ultrasonography of the temporal and ophthalmic arteries in the diagnosis and follow-up of giant cell arteritis. Clin Exp Rheumatol 2009; 27: $77-82$

[19] De Miguel E, Roxo A, Castillo C et al. The utility and sensitivity of colour Doppler ultrasound in monitoring changes in giant cell arteritis. Clin Exp Rheumatol 2012; 30 (70): S34-S38

[20] Schinkel AFL, van den Oord SCH, van der Steen AFW et al. Utility of contrast-enhanced ultrasound for the assessment of the carotid artery wall in patients with Takayasu or giant cell arteritis. Eur Heart J Cardiovasc Imaging 2014; 15: 541 - 546

[21] Bley TA, Uhl M, Carew J et al. Diagnostic value of high-resolution MR imaging in giant cell arteritis. AJNR Am J Neuroradiol 2007; 28: $1722-1727$

[22] Klink T, Geiger ], Both M et al. Giant cell arteritis: diagnostic accuracy of MR imaging of superficial cranial arteries in initial diagnosis-results from a multicenter trial. Radiology 2014; 273: 844 - 852

[23] Bley TA, Wieben O, UhI M et al. High-resolution MRI in giant cell arteritis: imaging of the wall of the superficial temporal artery. Am J Roentgenol 2005; 84: $283-287$

[24] Bley TA, Weiben O, Uhl M et al. Assessment of the cranial involvement pattern of giant cell arteritis with $3 \mathrm{~T}$ magnetic resonance imaging. Arthritis Rheum 2005; 52: 2470-2477

[25] Geiger ], Bley T, Uhl M et al. Diagnostic value of T2-weighted imaging for the detection of superficial cranial artery inflammation in giant cell arteritis. J Magn Reson Imaging 2010; 31 : $470-474$ 
[26] Bley TA, Markl M, Schelp M et al. Mural inflammatory hyperenhancement in MRI of giant cell (temporal) arteritis resolves under corticosteroid treatment. Rheumatology (Oxford) 2008; 47: 65-67

[27] Siemonsen S, Brekenfeld C, Holst B et al. 3T MRI reveals extraand intracranial involvement in giant cell arteritis. AJNR Am J Neuroradio 2015; 36: 91 - 97

[28] Salvarani C, Soriano A, Muratore F et al. Is PET/CT essential in the diagnosis and follow-up of temporal arteritis? Autoimmun Rev 2017; 16: $1125-1130$

[29] Soussan M, Nicolas P, Schramm C et al. Management of largevessel vasculitis with FDG-PET: a systematic literature review and meta-analysis. Medicine (Baltimore) 2015; 94 : e622. doi:10.1097/MD.0000000000000622
[30] Prieto-González S, Depetris M, García-Martínez A et al. Positron emission tomography assessment of large vessel inflammation in patients with newly diagnosed, biopsy-proven giant cell arteritis: a prospective, case-control study. Ann Rheum Dis 2014; 73: $1388-1392$

Bibliografie

DOI https://doi.org/10.1055/a-0831-0812

Dtsch Med Wochenschr 2019; 144: 587-594

(c) Georg Thieme Verlag KG, Stuttgart · New York ISSN 0012-0472

(ㄷ) (i) $(-)$ 